\title{
Robust Local Localization of a Mobile Robot in Indoor Environments Using Virtual Corners
}

\author{
Carlos Lara and Leonardo Romero \\ Universidad Michoacana, Morelia Mich. Mexico
}

\begin{abstract}
This paper deals with the problem of finding the movement of a mobile robot given two consecutive laser scans. The proposed method extracts a line map from the sequence of points in each laser scan, using a probabilistic approach, and then computes virtual corners between two lines in the same line map. The movement of the robot is estimated from correspondences of virtual corners between the two line maps. The combination of the probabilistic approach to find lines and the reduced number of virtual corners are the key ideas to get a simple, fast, robust to outliers, and reliable method to solve the local localization problem.
\end{abstract}

Keywords: Mobile Robotics, Local Localization, Line Based Map.

\section{Introduction}

A large number of today's mobile robots use a 2D laser range finder as a proximity sensor because it is fast and accurate. The range images given by a laser range finder can be used directly to perform simple tasks. However, most autonomous mobile robot tasks require that a map be built from the environment.

Normally a laser range finder takes measurements on a plane parallel to the floor and each laser scan provides $n$ points from the environment, with each point expressed in polar format $\left(\alpha_{i}, r_{i}\right), i=1 \ldots n$, where $r_{i}$ is the distance from the sensor to the detected object at direction $\alpha_{i}$.

The local localization problem consists of finding the relative pose of the robot. One way to solve the localization problem is to register two consecutive range images while the robot is moving, such as those shown in figure 1. In other words, we want to find the best parameters: translation and rotation of the robot $[\Delta x, \Delta y, \Delta \theta]$ that match sensed image (figure 10) and the reference image (figure 1 1 ). The problem of matching two images is known as image registration. An overview of techniques to solve image registration in computer vision is presented in [18.

There are two general methods to solve the registration problem: area-based methods and feature-based methods [18]. The feature-based methods are recommended if the images to be matched contain enough distinctive and easily detectable objects. The main idea relative to feature based-methods is to find invariant features in both images, find their correspondences, and then estimate the optimal transformation.

It is possible to apply the simple Iterative Closest Point (ICP) algorithm [2 directly to the raw points of the two consecutive range images. The ICP can

L. Rueda, D. Mery, and J. Kittler (Eds.): CIARP 2007, LNCS 4756, pp. 901-910 2007.

(C) Springer-Verlag Berlin Heidelberg 2007 


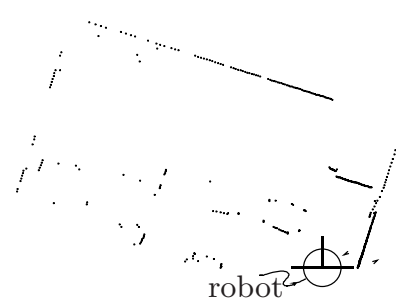

(a)

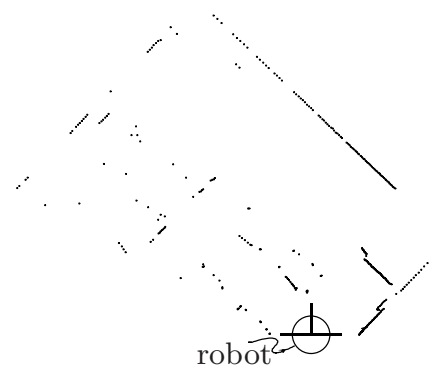

(b)

Fig. 1. Two consecutive Range Images

be considered as an area-based method because it uses raw data and does not extract any features from the data.

The ICP algorithm is specially effective when the robot has a good initial estimate of the post-move position. Unfortunately, without a good post-move position estimate, excessive processing time is required to achieve a good estimation. To solve the excessive processing time as well as including some information related to the environment, feature-based methods have been proposed. The kind of feature selected to resolve the image registration problem depends on the application. Many applications use lines, such as in [7]. For indoor mobile robots lines are useful because man-made environments are rich in planar objects. Lines also have a simple model and they are easy to detect.

Several features based on lines have been proposed to solve the laser scan matching problem in indoor environments: complete line segments [17, angles between consecutive or random lines [1, parallel or orthogonal lines [13, corners [9] or statistical information from the original raw data of the lines [6]. Other authors such as [11] [16] [15] select polylines instead of lines because polylines integrate information from several adjacent lines into a single feature. Polylines can be viewed as a generalization of lines or as an approximation of other types of curves.

This paper proposes a feature-based method to find the parameters of the rigid transformation $[\Delta x, \Delta y, \Delta \theta]$ between two range images provided by a laser scan mounted on a mobile robot. In order to do this, the method takes advantage of the angle invariance of any two lines along the point intersection of the lines, these information is merged in a single feature called virtual corner. It is important to note that a virtual corner can be produced by two adjacent lines or by any two lines. The method consists in two steps:

1. Feature detection. This step first learns a set of lines, called the line map, from both range images. This is done by using a probabilistic search to manage outliers. Then, from each line map a set of virtual corners are calculated, one per each line map. 
2. Feature matching. In this step every virtual corner of the first image is matched with one or more virtual corners of the second image. Every pair of virtual corners gives a set of parameters for the rigid transformation. To select the best set of parameters, an evaluation function measures the similarity between line maps using only the geometric parameters of their lines and the number of points associated with each line.

This approach has many advantages: it is simple, robust to noise, fast and reliable. In addition, feature detection is based on a probabilistic method to manage outliers. It also avoids the problem of estimate real corners (like 9]), specially when data are noisy, and it takes advantage of the intersection of any two lines. The proposed similarity measure is fast to compute and includes line comparisons and the number of points of each line.

The rest of the paper is organized as follows: section 2 describes the feature detection step and the section 3 describes the feature matching step. Section 4 shows some experimental results, and section 5 presents the advantages and drawbacks of the method and future work.

\section{Feature Detection}

Given a rigid transformation (translation and rotation only), the angle between any two lines, denoted by $\widehat{l_{1} l_{2}}$, is an invariant. See for instance lines in polar representation $l_{1}:\left(\alpha_{1}, \rho_{1}\right)$ and $l_{2}:\left(\alpha_{2}, \rho_{2}\right)$ shown in figure 2 2 . If lines $l_{1}^{\prime}$ and $l_{2}^{\prime}$ in figure $2 \mathrm{~b}$ correspond to $l_{1}$ and $l_{2}$ then $\widehat{l_{1} l_{2}}=\widehat{l_{1}^{\prime} l_{2}^{\prime}}$ or $\widehat{l_{1} l_{2}} \simeq \widehat{l_{1}^{\prime} l_{2}^{\prime}}$ for real situations where measurements are noisy. While other methods can use length ratios as an invariant, in the case of range images taken by a mobile robot some lines can be detected partially and the length ratio can differ drastically (specially when the robot approaches a door).

Virtual corners are based on the angle invariance of two lines, but also includes the intersection point of the lines. A virtual corner can correspond to a real corner of two adjacent line segments or an imaginary corner if the actual line segments are replaced by their lines. Even two parallel lines have a virtual corner located in the ideal or infinite point where parallel lines meet, see 8 for more information about infinite points. For further processing, a virtual corner of two lines $l_{1}$ and $l_{2}$ is represented by the intersection point $p_{12}$, the minor angle $\widehat{l_{1} l_{2}}$ between the lines, and their line's inclinations $\alpha_{1}$ and $\alpha_{2}$.

This section describes the process of finding virtual corners from a range image, this process is comprised of two steps: line map computation and virtual corner detection.

\subsection{Computing Line Maps}

There are several methods to calculate a set of lines, called line map, $\mathcal{M}=$ $\left\{l_{1} \ldots l_{m}\right\}$ from a sequence of points $\mathcal{P}=\left(p_{1} \ldots p_{n}\right)$, for an overview see [14. To manage problems associated with outliers (atypical data) it is preferable to use 


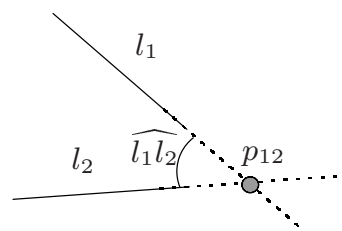

(a)

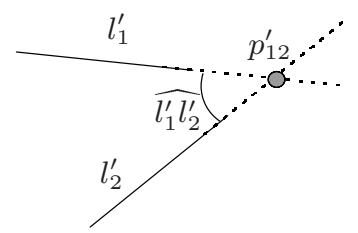

(b)

Fig. 2. Angle invariance between two range images

a robust method. We use the Window Sampled Consensus (WSAC) algorithm 12 because it is fast and gives good quality line maps. To extract a line the WSAC algorithm is divided in two stages:

Local Search. The local search stage looks for a line $l$ inside a set of $t_{l}$ consecutive points. As an example figure 3 a shows a window with $t_{l}=25$ consecutive points. To find the best local line $l$ this stage is based on the well-known RANSAC algorithm [5] and the M-Estimators [10]. If the algorithm successfully finds a local line $l$ then the global fit is performed.

Global Fit. This step looks for other points that fit with the line $l$, as shown in figure 3b. The global search is comprised by three steps: First the algorithm determines the set of points that support line $l$ by searching into the whole laser scan $\mathcal{P}$. Second, the points that belong to small length segments are removed. This step finds the segments by applying a Breakpoint Detector algorithm similar to the one presented in [3. Finally the line parameters are recomputed using the set of inliers and the line is added to the map $\mathcal{M}$. Figure $3 \mathrm{~b}$ shows the line $l$ after the global fit, it has three segments: $s_{1}, s_{2}$ and $s_{3}$.

An important consideration is that after the global fit the points that belong to $l$ are removed from the laser scan $\mathcal{P}$. The WSAC algorithm tries iteratively the local search step and global fit step to find the line map $\mathcal{M}$.

\subsection{Detecting Virtual Corners}

Given the line map $\mathcal{M}=\left\{l_{1} \ldots l_{n}\right\}$, a set of virtual corners is calculated by intersecting any two lines $l_{i}, l_{j} \in \mathcal{M}$. Virtual corners that have point intersection at infinity or at some point far away are removed from the map. The number of possible virtual corners is $O\left(n^{2}\right)$.

\section{Feature Matching}

In this step, the correspondence between the virtual corners detected in the sensed image and those detected in the reference image is established. For this purpose, every virtual corner in the sensed image is compared against virtual corners in the reference image. Only pairs of virtual corners having similar angles 


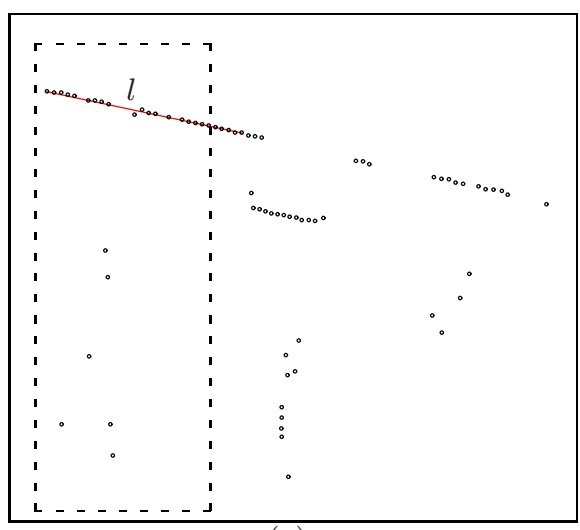

(a)

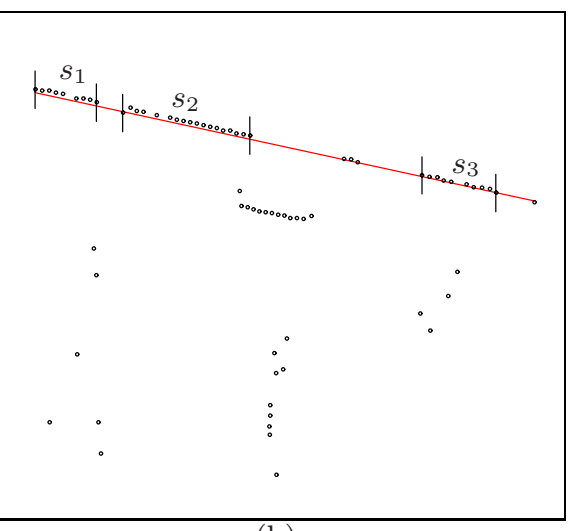

(b)

Fig. 3. WSAC Algorithm

are selected. Every pair of virtual corners give a putative set of parameters $[\Delta \theta, \Delta x, \Delta y]$. Given two virtual corners from the reference and the sensed images as the shown in $4 \mathrm{a}$ and $4 \mathrm{~b}$, the parameter $\Delta \theta$ is calculated from two lines (for instance $l_{2}$ and $l_{2}^{\prime}$ in figure 4 ; ), then parameters $\Delta x$ and $\Delta y$ are also calculated by aligning virtual corners as shown in figure $4 \mathrm{~d}$. Other putative parameters can be calculated by aligning two different lines, for instance $l_{1}$ and $l_{2}^{\prime}$. For reducing the complexity and avoiding incorrect estimations the set of parameters must fulfil some requirements, as an example, $|\Delta \theta|<t_{\theta}$ and $\sqrt{(\Delta x)^{2}+(\Delta y)^{2}}<t_{t}$, where $t_{\theta}$ and $t_{t}$ are thresholds that depends on the maximum rotation and translation expected of the robot.

To select the best set of parameters, line maps $\mathcal{M}$ and $\mathcal{M}^{\prime}$ associated to reference and sensed image respectively, must be compared. To compare them the first step is to transform the line map $\mathcal{M}^{\prime}$ into $\mathcal{M}_{T}^{\prime}$, that is, for each line $l^{\prime} \in \mathcal{M}^{\prime}$ new parameters $\left(\alpha_{T}^{\prime}, \rho_{T}^{\prime}\right)$ are calculated considering the putative transformation $T:[\Delta \theta, \Delta x, \Delta y]$. This is done by using:

$$
\begin{aligned}
\alpha_{T}^{\prime} & =\alpha^{\prime}+\Delta \theta \\
\rho_{T}^{\prime} & =\rho^{\prime}+\Delta x \cos \alpha^{\prime}+\Delta y \sin \alpha^{\prime}
\end{aligned}
$$

To evaluate the similarity $S$ between $\mathcal{M}$ and $\mathcal{M}_{T}$, we propose the following function;

$$
S\left(\mathcal{M}, \mathcal{M}_{T}\right)=\sum_{\substack{l \in \mathcal{M} \\ l \\ l \\ l_{T}^{\prime} \in \mathcal{M}_{T}^{\prime}}} s\left(l, l_{T}^{\prime}\right) \cdot \min \left(\mathrm{w}, \mathrm{w}^{\prime}\right)\left(1-\frac{\left|\mathrm{w}-\mathrm{w}^{\prime}\right|}{\max \left(\mathrm{w}, \mathrm{w}^{\prime}\right)}\right)
$$

where $w$ and $w^{\prime}$ are the number of inliers of $l$ and $l_{T}^{\prime}$ respectively and $s\left(l, l_{T}^{\prime}\right)$ is a similarity value between any two lines $l \in \mathcal{M}, l_{T}^{\prime} \in \mathcal{M}_{T}^{\prime}$. The measure of similarity between lines was taken from [4]: 


$$
s\left(l, l_{T}^{\prime}\right)=\frac{1}{1+(\Delta \alpha)^{2} * \alpha_{0}+(\Delta \rho)^{2} * \rho_{0}}
$$

where $\Delta \alpha=\alpha-\alpha^{\prime}, \Delta \rho=\rho-\rho^{\prime}$, and $\alpha_{0}, \rho_{0}$ are constants. The optimal transformation $T^{*}$ is retrieved as the transformation $T$ which gives the highest value $S\left(\mathcal{M}, \mathcal{M}_{T}^{\prime}\right)$.

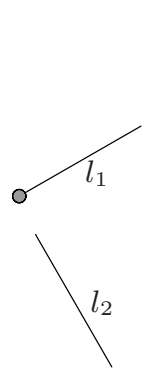

(a)

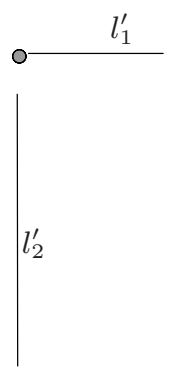

(b)

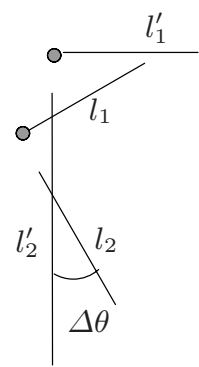

(c)

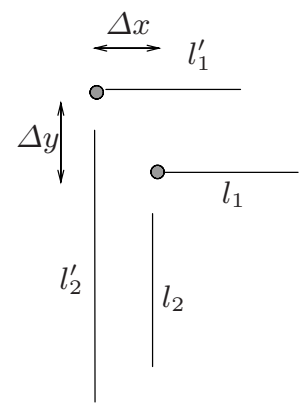

(d)

Fig. 4. Aligning two virtual corners. a) A corner in the reference image, b) on the sensed image, c) finding $\Delta \theta, \mathrm{d})$ finding $\Delta x, \Delta y$.

\section{Experimental Results}

The algorithm described above was tested in a set of range images taken by our real mobile robot equipped with a LMS209-S02 SICK Laser Measurement System. Each laser scan covers $180^{\circ}$ with a lateral resolution of $0.5^{\circ}$, a total of 361 points per laser scan. The values used for $t_{\theta}$ and $t_{t}$ were $\frac{\pi}{2}$ and $1 \mathrm{~m}$ respectively.

Figure 5 shows an example of the proposed method using range image 1 and range image 2 . The final match achieves the desired result and so the translation of the robot was accurately estimated.

Figure 6 shows a more complex example where the robot rotates and so each laser scan see different parts of the environment. In other words, there are some points in one laser scan which do not correspond to any points in the other laser scan. The final match achieves the desired result and so the rotation of the robot was accurately estimated.

Table 1 shows a summary of the test performed considering 50 movements of the robot, translations $(50 \mathrm{~cm})$ and rotations $\left( \pm 25^{\circ}\right)$. This table shows the average number of lines detected per laser scan, the average number of virtual points, the total number of virtual corner correspondences, and finally the actual number of correspondences which were evaluated using the similarity measure. As the reader can see, there is a significant amount of correspondences that are not evaluated, because they do not have a similar angle (first filter) or they give translations or rotations greater than $t_{t}$ or $t_{\theta}$ (second filter). 


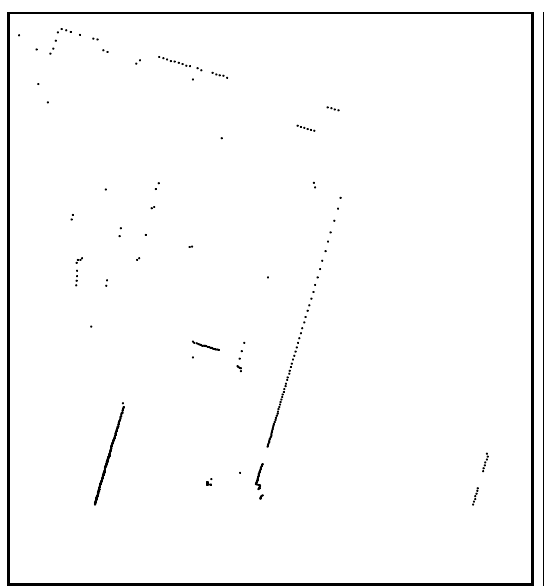

(a)

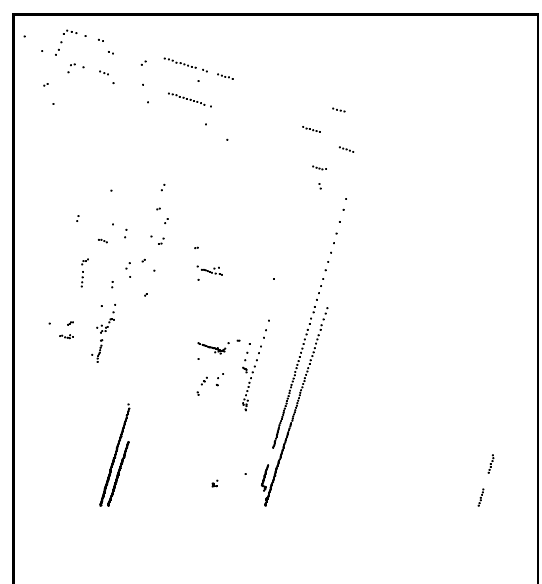

(c)

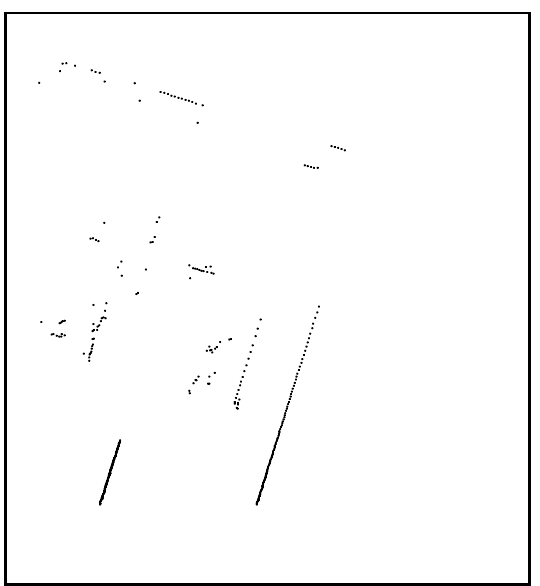

(b)

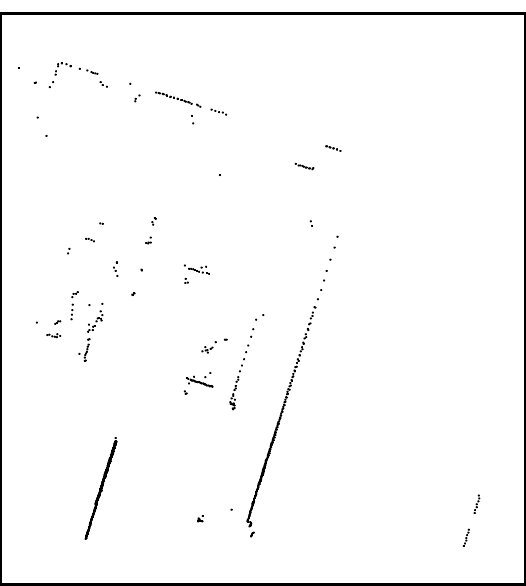

(d)

Fig. 5. The robot moves forward: (a) Range image 1, (b) Range image 2, (c) overlapped images with no robot movement and (d) Final match

\section{Conclusions and Future Work}

Local localization is one of the most important tasks for mobile robots. One way to solve the local localization problem is the registration of consecutive range images provided by a laser range finder. This paper proposes a method that uses virtual corners to solve the localization problem and it does not require an odometric estimation. A virtual corner is a feature that includes geometric information of any two lines discovered in range images. The results show that the method is fast and reliable but requires that almost two reliable virtual corners being discovered: one from the reference image and their equivalent from the sensed image. 


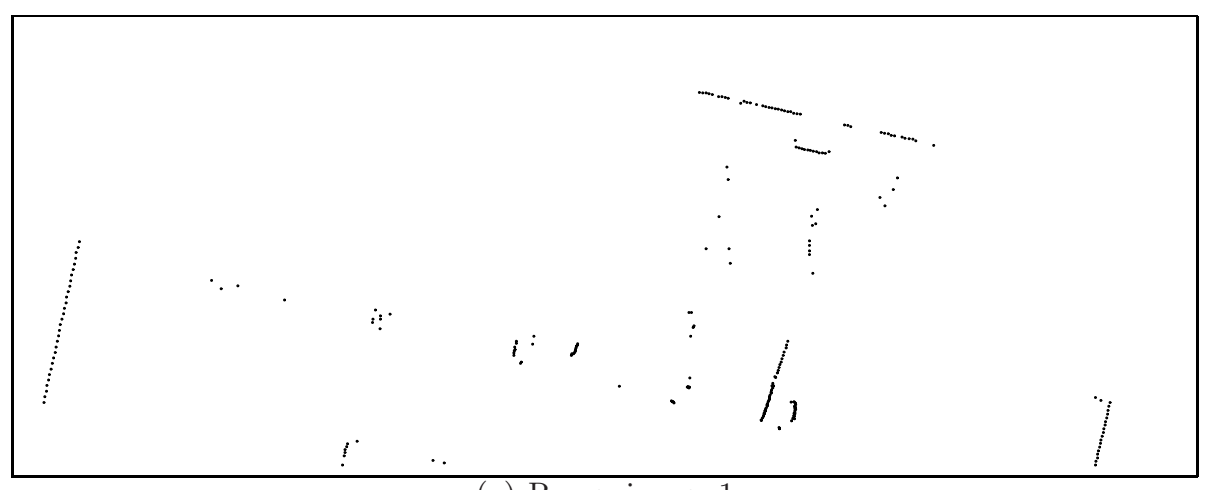

(a) Range image 1

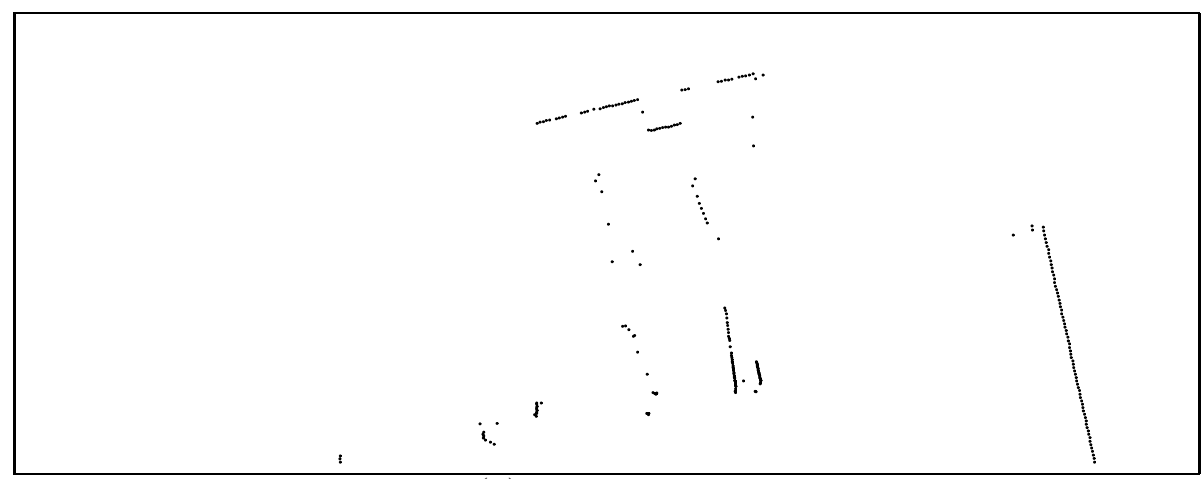

(b) Range image 2

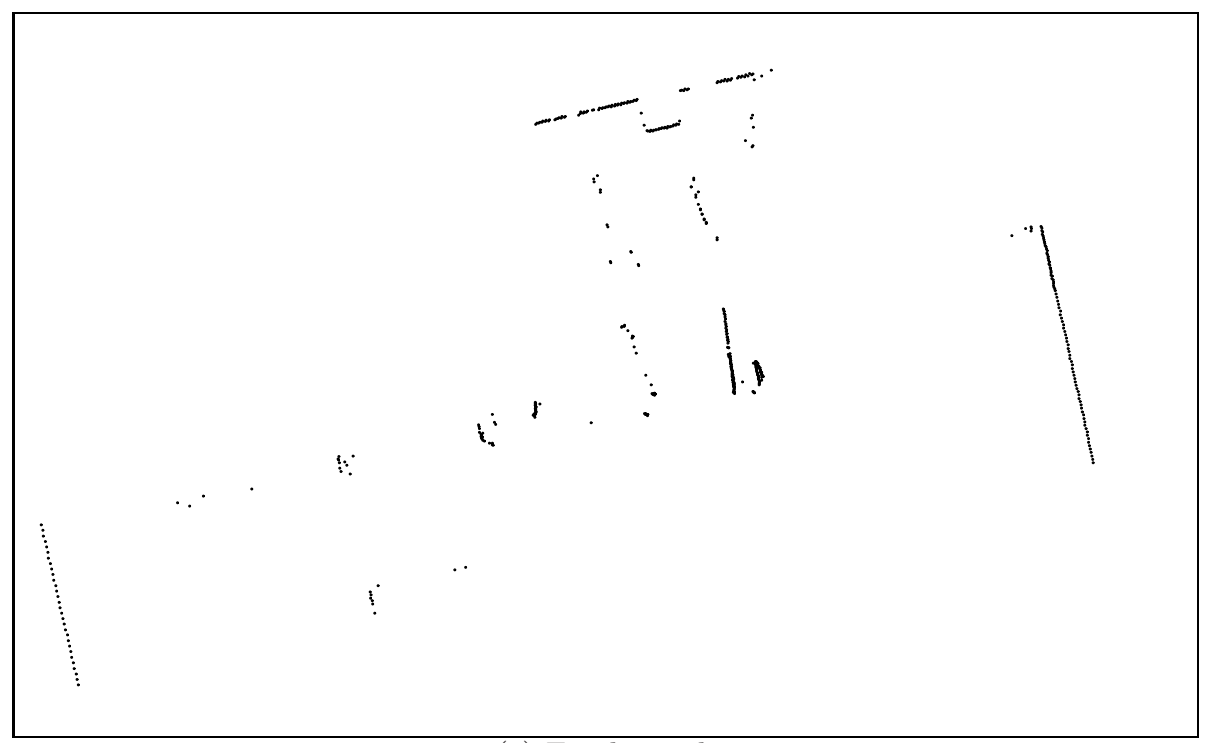

(c) Final match

Fig. 6. Local localization when the robot rotates 
Table 1. Results considering 50 movements (translations and rotations) of a mobile robot

\begin{tabular}{|c|c|}
\hline Parameter & Average \\
\hline \hline Lines detected & 10.92 \\
Virtual Points & 118.36 \\
Correspondences & 15148.96 \\
Evaluated Correspondences & 160.10 \\
\hline
\end{tabular}

In the near future we are going to include: i) a robust line estimator in the global fit stage, ii) a new filter to reduce the number of correspondence evaluations, for instance clustering results, iii) a new probabilistic similarity measure based on the points supporting lines, and iv) more features such as single points. The goal is to improve the accuracy of the results and the method could be applied to solve the global localization and mapping problems.

\section{References}

1. Amigoni, F., Gasparini, S., Gini, M.: Building segment-based maps without pose information. Proceedings of the IEEE 94(7), 1340-1359 (2006)

2. Besl, P.J., Mckay, N.D.: A method for registration of 3-d shapes. IEEE Transactions on Pattern Analysis and Machine Intelligence 14(2), 239-256 (1992)

3. Borges, G.A., Aldon, M.-J.: Line extraction in $2 \mathrm{~d}$ range images for mobile robotics. J. Intell. Robotics Syst. 40(3), 267-297 (2004)

4. Einsele, T.: Localization in indoor environments using a panoramic laser range finder. PhD thesis, Technische Universit at Munchen (2001)

5. Fischler, M.A., Bolles, R.C.: Random sample consensus: a paradigm for model fitting with applications to image analysis and automated cartography. Commun. ACM 24(6), 381-395 (1981)

6. Garulli, A., Giannitrapani, A., Rossi, A., Vicino, A.: Simultaneous localization and map building using linear features. In: Proceedings of the 2nd European Conference on Mobile Robots (2005)

7. Gros, P., Bournez, O., Boyer, E.: Using local planar geometric invariants to match and model images of line segments. Comput. Vis. Image Underst. 69(2), 135-155 (1998)

8. Hartley, R.I., Zisserman, A.: Multiple View Geometry in Computer Vision, 2nd edn. Cambridge University Press, Cambridge (2004)

9. Hernandez-Alamilla, S., Morales, E.: Global localization of mobile robots for indoor environments using natural landmarks. In: IEEE Conference on Robotics Robotics, Automation and Mechatronics, pp. 1-6 (2006)

10. Huber, P.: Robust Statistics. Wiley, New York (1981)

11. Lakmper, R., Latecki, L.J., Sun, X., Wolter, D.: Geometric robot mapping. In: DGCI, pp. 11-22 (2005)

12. Lara, C., Romero, L.: A robust approach to build 2 d line maps from laser scans. Advances in Computer Science and Engineering. Research in Computing Science 19, 197-208 (2006) 
13. Nguyen, V., Harati, A., Tomatis, N., Martinelli, A., Siegwart, R.: Orthogonal slam: a step toward lightweight indoor autonomous navigation. In: Proc. of the IEEERSJ Int. Conf. on Intelligent Robots and Systems (2006)

14. Sack, D., Burgard, W.: A comparison of methods for line extraction from range data. In: IAV (2003)

15. Veeck, M., Burgard, W.: Learning polyline maps from range scan data acquired with mobile robots. In: IROS (2004)

16. Wolter, D., Latecki, L.J.: Shape matching for robot mapping. In: Zhang, C., W. Guesgen, H., Yeap, W.-K. (eds.) PRICAI 2004. LNCS (LNAI), vol. 3157, pp. 693702. Springer, Heidelberg (2004)

17. Zezhong, X., Jilin, L., Zhiyu, X.: Map building and localization using 2d range scanner. In: Proceedings 2003 IEEE International Symposium on Computational Intelligence in Mobile Robotics and Automation, pp. 848-853 (2003)

18. Zitova, B., Flusser, J.: Image registration methods: a survey. Image and Vision Computing 21(11), 977-1000 (2003) 